\title{
Too Much Of A Good Thing? A Review Of Volatility Extensions In Black-Scholes
}

\author{
Lamya Kermiche, Grenoble Ecole de Management, France
}

\begin{abstract}
Since the seminal Black and Scholes model was introduced in the 1970s, researchers and practitioners have been continuously developing new models to enhance the original. All these models aim to ease one or more of the Black and Scholes assumptions, but this often results in a set of equations that is difficult if not impossible to use in practice. Nevertheless, in the wake of the financial crisis, an understanding of the various pricing models is essential to calm investors' nerves. This paper reviews the models developed since Black and Scholes, giving the advantages and disadvantages of each type. It focuses on the main variable for which Black and Scholes gives results that differ widely from market data: implied volatility. This variable also forms the basis for the development of a new type of models, called "market models."
\end{abstract}

Keywords: Black-Scholes Model; Implied Volatility; Market Models

\section{INTRODUCTION}

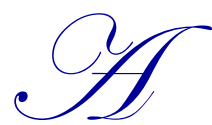

few years ago, the financial markets struggled through one of the biggest crises in decades. This period has been characterized by the highest levels of volatility ever observed on the markets, with the CBOE Volatility Index (VIX) reaching a level of $80.6 \%$ on October $27,2008 .{ }^{1}$ Derivatives have been particularly hard-hit as investors begin to wonder whether such instruments are indeed sound investments. A lot of the suspicion about derivatives stems from a lack of understanding as well as a broad distrust of the risk management systems meant to keep such instruments in check.

These risk management systems, as well as the associated pricing methods, are based on complex mathematical models. The first model was developed by Black and Scholes in the early 1970s, and models have been getting increasingly intricate ever since. Black-Scholes marked the beginning of a rapid expansion in derivatives markets, as it provides a fairly straightforward method for pricing and hedging derivatives based on various risk factors. The main drawback to Black-Scholes is that it is based on simplistic assumptions such as constant volatility and a normal distribution function for the underlying asset return. This can lead to substantial discrepancies between actual market prices and prices calculated using the model.

These discrepancies between market and theoretical prices are most evident in the observation of different implied volatilities according to exercise prices (smile or skew) and maturities (term structure). Several empirical studies have confirmed this phenomena, including those by Rubinstein (1994) and Dumas, Fleming, and Whaley (1998). The implied volatility surface is not flat and varies with time. Implied volatility has subsequently become a preferred variable among traders who often quote options in terms of volatility rather than price.

This paper compares the main modelling advancements made since Black-Scholes, highlighting the advantages and disadvantages of each one. Skiadopoulos (2001) has presented a literature review for pricing models that are consistent with the volatility smile. While extending his work, our approach is unique in that we also consider a new type of models — known as "market models" — based on modelling directly implied volatilities.

This paper is structured as follows: Section 2 outlines the various approaches that have been developed to enhance the Black-Scholes model. Section 3 discusses implied volatility more precisely. Implied volatility also

\footnotetext{
${ }^{1}$ Source: Chicago Board of Options Exchange
} 
forms the basis for the development of market models, which are becoming increasingly popular. Before the concluding section, a synthesis of the findings is presented, along with the most promising venues for future research.

\section{ALTERNATIVES TO THE BLACK-SCHOLES MODEL}

Options theory is used to price derivatives and set up hedging strategies to cover the associated risks. It was initiated by Black and Scholes (1973), who developed closed-form formulas to calculate the prices of European Call and Put options, based on certain assumptions. For instance, according to Black-Scholes, the price of the underlying asset $S$ follows a lognormal process, with an expected return $\mu$ and a constant volatility $\sigma$. The diffusion process is thus of the form:

$\frac{d s_{t}}{s_{t}}=\mu d t+\sigma d W_{t}$

where $\mathrm{W}_{\mathrm{t}}$ is a standard Brownian process

We now know that these assumptions are not realistic. Researchers have developed several alternatives to the Black-Scholes model in order to account for implied volatility smiles, and for skewness and kurtosis in the distribution of the returns. These alternative models can be divided into three categories, where each category modifies the original model in order to ease some of its constraints. The first category consists of models with a jump diffusion process, and was introduced by Merton in 1976 - not long after Black and Scholes published their work. The second category appeared in the 1980s, and includes stochastic volatility models; the main models in this category were developed by Hull and White (1987), Stein and Stein (1991), and Heston (1993). The third category comprises local volatility models, which were developed primarily by Dupire (1994), Derman and Kani (1994a), and Rubinstein (1994). Researchers have also studied improvements to and combinations of these categories.

The following sections describe each of these categories and give the benefits and limitations of each one, as well as proposed enhancements.

\subsection{Jump Diffusion Models}

It is Merton (1976) who introduced the idea of adding a jump component (following a Poisson process) to the Black-Scholes diffusion process. This makes economical sense since markets receive new information in a discrete manner, and instantly adjust the underlying asset price accordingly. Therefore the addition of a jump component makes the model more representative of actual market conditions by accounting for outliers and for the asymmetry of the return distribution curve. The equation for the underlying asset diffusion process thus becomes:

$$
\frac{d S}{S}=\mu d t+\sigma d W+d q
$$

where $d q$ is the jump component and follows a Poisson process

Several studies, including those by Ball and Torous (1985), Das and Uppal (1998), and Das (2002), tested Merton's model and, after estimating values for its parameters, found that the jump component is significant. The benefit of this type of model is that, depending on the parameter values used, it can generate several different volatility smile and skew shapes according to what is seen in the markets. For instance, using a jump process with a negative average gives the sharp skew seen for short-term maturities. Das and Foresi (1996), Bates (1996), and Bakshi, Cao, and Chen (1997) have illustrated the importance of the jump component for pricing very short-term options. For instance, Bakshi, Cao, and Chen (1997) compared several models and concluded that jumps are essential for modelling the underlying asset diffusion process when pricing short-term options, even if using a stochastic volatility model (discussed below). Models with jumps also better account for the skewness and kurtosis in the distribution of returns. 
However, models with jumps are difficult to put into practice because they do not offer a simple equation to calculate options prices. Another significant limitation, highlighted by Andersen, Benzoni, and Lund (2002), is that although these models give an implied volatility smile consistent with actual observations, they do not account for changes in the level and shape of the implied volatility smile over time. Implied volatility smile dynamics is one of the main factors covered by more recent options pricing models, as discussed in the next section.

\subsection{Stochastic Volatility Models}

Stochastic volatility models form the second alternative to Black-Scholes. In these models the underlying asset volatility is considered stochastic and its movements are represented by a diffusion process. Hull and White (1987) were among the first to study this type of model, and were soon followed by Stein and Stein (1991), Heston (1993), among others.

In these models, the underlying asset diffusion process is still considered lognormal, as given by Equation (1), but the volatility $\sigma$ is not constant. It also follows a stochastic process, which can be correlated with the underlying asset diffusion process. Therefore a two-equation system is needed to model the underlying movements.

Researchers have proposed several types of stochastic processes to model the volatility. For example, Hull and White (1987) suggest that the variance (volatility squared) also follows a lognormal process, given by:

$\frac{d \sigma^{2}}{\sigma^{2}}=\delta d t+\xi d Z$

where $\delta$ and $\xi$ represent, respectively, the drift and the volatility of the volatility. Unfortunately, solving this twoequation system with partial derivatives requires complex numerical methods. Stein and Stein (1991) developed another model where a mean reversion process is used for the volatility diffusion process. This makes economical sense since volatility can neither increase nor decrease indefinitely. Their equation for the volatility diffusion process is:

$d \sigma=\delta(\theta-\sigma) d t+\xi d Z$

They subsequently developed a semi-analytical equation for pricing options, but they assumed that the volatility is not correlated with the underlying asset - which doesn't allow accounting for skewness. Indeed, Bakshi, Cao, and Chen (1997) indicate that in stochastic volatility models, the correlation between the volatility and the underlying asset determines the level of skewness, while the volatility of the volatility determines the level of kurtosis in the distribution of the underlying asset.

Heston (1993) relaxed Stein and Stein's model by allowing volatility to be correlated to the underlying asset; in his version, the variance follows a mean reversion function as follows:

$d \sigma^{2}=\delta\left(\theta-\sigma^{2}\right) d t+\xi \sigma d Z$

Heston consequently developed a semi-analytical equation that can be solved by using an inverse Fourier transform. The advantage of stochastic volatility models is that they give different smile and skew shapes depending on the level of the correlation, and the parameters used for the volatility process. For example, a zero correlation gives a pure smile whose level depends on the volatility of the volatility; a negative correlation gives a skew (volatility decreases as the exercise price increases); and a positive correlation gives the opposite effect (volatility increases as the exercise price increases).

Nevertheless, these models have their share of limitations. First, an unrealistic (extremely negative) correlation between the volatility and the underlying asset must be used to obtain a skew consistent with what is seen in the markets. For instance, Taylor and Xu (1993) found that the smile calculated using a stochastic volatility model with reasonable parameters is half of what is actually observed. Second, stochastic volatility models are multi-factor models that are difficult to use and cannot be employed for dynamic hedging. Third, these models are 
not very effective at pricing short-term options unless an unrealistic value is used for the volatility of the volatility (see Bakshi, Cao, \& Chen, 1997).

Stochastic Volatility and Jumps: Some researchers, including Bates (1996), Bakshi, Cao, and Chen (1997), and Andersen, Benzoni, and Lund (2002), have added a jump component to stochastic volatility models so as to merge the best elements of the two model types. They compared stochastic volatility models with and without jumps, and found that it is very important to account for the stochastic volatility - but that requires using improbable values for correlation and volatility of the volatility to represent the skewness and kurtosis in the distribution of the returns. Adding a jump component improves the model substantially, especially for short-term options.

Therefore both elements-jump components and stochastic volatility processes-are needed to appropriately represent market data, and to price options. A negative correlation between the volatility and the underlying asset must also be used in order to obtain the implied volatility skew. Note also that Andersen, Benzoni, and Lund (2002) did not use options to estimate the parameter values of their jump-augmented stochastic volatility model; their work then showed that the jump component, as well as the volatility's stochastic nature and its negative correlation with the underlying asset are intrinsic features of the underlying asset movements.

Finally, Bates (1996), Bakshi, Cao, and Chen (1997), and Andersen, Benzoni, and Lund (2002) all concluded that any reasonable representation of the underlying asset price diffusion must incorporate both a stochastic volatility and a jump diffusion process.

\subsection{Local Volatility Models}

The introduction of jumps (Merton, 1976) and stochastic volatility (Hull \& White, 1987) make the model no longer complete; indeed, since only the underlying asset (and not the volatility) is a traded asset, the market itself is incomplete. But completeness is a central concept in options pricing and arbitrage-free hedging.

According to Schönbucher (1999), stochastic volatility models can reproduce the implied volatility curve shapes typically seen in the markets (smile and skew), but cannot easily be calibrated with any implied volatility surface. Moreover, they do not give a simple equation for pricing standard options.

Research into a model that can be easily calibrated from the observed implied volatility surface at a given moment led to the development of local volatility models. These models provide a simple way to price options using implied trees. There are currently two types of local volatility models: deterministic local volatility models and stochastic local volatility models, each of which is described below.

\subsubsection{Deterministic Local Volatility Models}

Dupire (1993a, 1994) initiated research into deterministic local volatility models. He removed the constant volatility assumption and introduced the local volatility theory, whereby the instantaneous volatility is considered to be a deterministic function of time and the underlying asset. This gives the following equation for the underlying asset diffusion process:

$\frac{d S_{t}}{s_{t}}=\mu d t+\sigma\left(t, S_{t}\right) d W_{t}$

The local volatility $\sigma_{L}\left(t, S_{t}\right)$ is equal to the instantaneous volatility $\sigma\left(t, S_{t}\right)$ at a future time $t$ and corresponding underlying asset value $S_{t}$, so that all observed options are priced in a consistent manner. Dupire showed that the local volatility function can be uniquely determined from a market price surface $C(T, K)$ of standard European options with different exercise prices and maturities. This led to the famous Dupire formula:

$\sigma_{L}^{2}\left(t, S_{t} \mid t=T, S_{t}=K\right)=2 \frac{\frac{\partial C}{\partial T}+(r-q) K \frac{\partial C}{\partial K}+q C}{K^{2} \frac{\partial^{2} C}{\partial K^{2}}}$ 
Once the local volatility function has been determined, the model can be used to calculate future underlying asset prices. Therefore any option can be priced using this diffusion process, and the resulting price will be consistent with all liquid options on the same underlying asset (standard European options). Dupire also suggested using trinomial trees to make this process a discrete process.

Meanwhile, Derman and Kani (1994a) and Rubinstein (1994) developed a similar approach using binomial trees. Derman, Kani, and Chriss (1996) subsequently extended this approach to implied trinomial trees, which are more flexible than binomial trees. The advantage of the latter approach is that the local volatility can be calculated from the smile without needing to make any assumptions about its shape.

Many traders use the local volatility approach because it is both easy to use (thanks to the implied binomial and trinomial trees) and retains the market completeness of the Black-Scholes model. However, Andersen and Andreasen (2000) have criticised this approach, claiming that movements in the smile in a deterministic local volatility model lead to highly unstationary implied volatilities, and indicate that the implied volatility skew will disappear in the near future-which contradicts what is seen in empirical observations. Andersen and Andreasen therefore extended deterministic local volatility models to account for jumps in the underlying asset diffusion process. Their model brings together the best features of both model types; the jump process allows for the modelling of steep short-term skews, and the local volatility approach ensures that the model is in line with market options prices.

We should also consider another important point: binomial and trinomial trees account for the overall implied volatility structure seen in the markets, but they cannot reflect dynamic changes in this structure. This is why they perform so poorly in dynamic asset management. For example, Dumas, Fleming, and Whaley (1998) tested the predictive and hedging power of a deterministic local volatility model (with a parametric local volatility surface) in dynamic asset management, and found that it performed no better than the simple Black-Scholes model. This led to the extension of these deterministic local volatility models to account for the stochastic nature of the local volatility, as discussed below.

\subsubsection{Stochastic Local Volatility Models}

Due to the dynamic hedging limitations of deterministic local volatility models, several researchers sought to enhance them, including the developers of these models themselves - who often proposed extensions to their own models. Dupire (1993b, 1996) added a stochastic dynamic to the volatility term structure to model the realised volatility. Derman and Kani (1998) extended their implied tree approach by adding a stochastic dynamic to the total local volatility surface (term structure and smile). They derived restrictions on local volatility trends in order to exclude any arbitrage opportunities. However these researchers have themselves admitted that their models contain complicated conditions and are difficult to use.

More recently, Alexander and Nogueira (2004) pointed out that the deterministic local volatility assumption implies that the instantaneous volatility is also deterministic - but this is inconsistent with numerous empirical studies that have proved the importance of stochastic volatility models. Therefore Alexander and Nogueira developed a stochastic local volatility model to merge these two model types. Although the local volatility surface may occasionally (at each implied tree calibration) be a deterministic function of $t$ and $S$, the surface changes with time in a stochastic manner. They came up with the following equation for the underlying asset diffusion process:

$\frac{d S_{t}}{S_{t}}=\mu d t+\sigma\left(t, S_{t} ; v_{1}, \ldots, v_{n}\right) d W_{t}$

The functions $v(t)=\left(v_{l}(t), \ldots, v_{n}(t)\right)$ are those of a deterministic local volatility model; they are stochastic functions correlated with each other and with the underlying asset. Therefore the instantaneous volatility $\sigma\left(t, S_{t}\right.$; $v(t))$ is also stochastic.

Similarly, Carmona and Nadtochiy (2009) propose a model based on the prescription of stochastic dynamics for the local volatility surface, and characterize the absence of arbitrage by means of a drift and a spot consistency conditions. 
However, as stated by Alexander and Nogueira (2004), these models work just as well as the implied volatility market models developed by Schönbucher (1999) in terms of hedging performance. The next section looks more closely at implied volatility and Schönbucher's market models.

\section{THE IMPLIED VOLATILITY SURFACE}

The past few years have seen a growing interest in implied volatility as an exogenous variable. This interest stems from the emergence of models in which implied volatility is considered as a state variable with a well-studied diffusion process. These models, called market models, assume that there is a sufficient number of standard options traded on the markets, and that these options can be used to price illiquid and exotic options in a way that is consistent with the smile.

Modelling implied volatility rather than instantaneous volatility (as is done in traditional stochastic volatility models) has many advantages. First, implied volatility can be observed directly in the markets - unlike instantaneous and local volatility - using options prices and without having to make any assumptions about the process. The diffusion process parameters are easier to estimate, especially the volatility of the implied volatility parameter. Second, options pricing models obtained by modelling implied volatility lead to prices that are automatically in adequacy with standard options prices seen in the markets. The implied tree models initially developed by Dupire (1993a, 1994), Derman and Kani (1994a, 1994b), and Rubinstein (1994) also have this benefit, but they do not account for the stochastic nature of implied volatility surfaces. Third, implied volatility models can easily be extended to multi-factor models.

One advantage relevant to traders is that traders are more familiar with implied volatility, and use it as a market risk indicator. They often quote options in terms of their implied volatility, since this requires fewer updates than quoting the price itself.

Finally, implied volatility is increasingly recognised by markets as an independent variable, as seen in the newly-introduced implied volatility indices and their associated derivatives. One possible drawback to this type of modelling is that there is not a single implied volatility value, but an entire implied volatility surface. Therefore all implied volatilities must be accounted for at the same time, which could quickly lead to systems with highdimension problems. However, Cont and Da Fonseca (2002) noted that the implied volatility surface represents options on the same underlying asset, and are linked by non-arbitrage relationships; the implied volatilities for different exercise prices and maturities are highly correlated and do not move independently. This suggests that the implied volatility surface dynamic can be modelled by just a few factors.

Thus a study of implied volatility behaviour - including the number and form of factors representing the implied volatility surface dynamic - is essential to developing a market model. The next section discusses the various market models presented in the literature as well as the resulting non-arbitrage conditions. This is followed by a review of empirical studies of the implied volatility surface dynamic.

\subsection{Implied Volatility Market Models}

Schönbucher (1999) was the first to develop a stochastic implied volatility model. He was followed by several others, including Brace, Goldys, Klebaner, and Womersley (2001), Ledoit, Santa-Clara, and Yan (2002), Cont, Da Fonseca, and Durrleman (2002), Le (2005), Daglish, Hull, and Suo (2007), and more recently Kallsen and Krühner (2014). Their work was motivated by the explosion in the derivatives market in the last decades. Indeed, the significant advancement represented by the Black-Scholes model, and the creation the same year of the first options market in Chicago, spurred an exponential growth in the volume and liquidity of traded options. Moreover, traders began using increasingly complex options (barrier options, Asian options, etc.).

Thus on the one hand options were becoming increasingly complex, and on the other standard European and American options were becoming increasingly liquid. Schönbucher (1999) noted that these developments gave researches valuable new information to study volatility. This information is crucial for pricing options but cannot be observed from the underlying asset price. Cont and Da Fonseca (2002) pointed out that since derivative markets 
have been operating with increasing independence, options prices depend not only on movements in the underlying asset but also on the specific features of these markets. For example, Bakshi, Cao, and Chen (2000) showed that models based on a single diffusion process ${ }^{2}$ are not consistent with observed changes in options prices. They concluded that options are not redundant instruments (i.e., they cannot be replicated with the underlying asset and a risk-free asset). Along the same lines, Fleming, Ostdiek, and Whaley (1995) showed that underlying asset volatility predictions based on the implied volatilities of options are more reliable than those obtained from the underlying asset process alone. Therefore it is clear that options contain information that is not in the underlying asset price and this information should be taken into account.

Implied volatility models aim to incorporate the information available from standard options, using these options' liquidity to price and hedge more complicated and less liquid options. More specifically, they aim to factor the randomness inherent to derivatives markets, which cannot be found in the underlying asset. Implied volatility is considered to move in a stochastic manner depending on numerous noise sources, possibly correlated with the process that generates the underlying asset price.

In these models the underlying asset process is represented by a lognormal function with stochastic volatility $\sigma$, as follows:

$\frac{d S_{t}}{S_{t}}=\mu d t+\sigma\left(t, S_{t}\right) d W_{t}$

For a given exercise price $K$ and maturity $T$, the implied volatility diffusion process is generally given by:

$d \sigma_{i m p}(T, K)=u(T, K) d t+\gamma(T, K) d W_{t}+\sum_{i=1}^{N} v_{i}(T, K) d Z_{i}$

The implied volatility thus derives from the Brownian motions $d Z_{i}$, and $d W_{t}$; the latter Brownian motion is the same as the one used to represent the underlying asset dynamic. $\gamma(T, K) d W_{t}$ represents the correlation between the underlying asset and the implied volatility (we typically see a negative correlation).

Schönbucher (1999) uses the opposite approach from that used in stochastic volatility models. Whereas stochastic volatility models specify the instantaneous volatility diffusion process and subsequently derive options prices and implied volatilities, Schönbucher specifies the implied volatility process and derives a consistent process for the instantaneous volatility. Schönbucher's approach seems to be more pragmatic since instantaneous volatilities cannot be observed in the market.

Brace, Goldys, Klebaner, and Womersley (2001), Ledoit, Santa-Clara, and Yan (2002), and Zilber (2007) developed models similar to Schönbucher's; the only difference is the function used to represent the volatility surface. Schweizer and Wissel (2008a, 2008b) generalised Schönbucher's work to other payoff functions. Le (2005) does not make any assumptions about the underlying asset process. Kallsen and Krühner (2014) adapt the HeathJarrow-Morton approach on interest rates to stock options, using time-inhomogeneous Lévy processes. All of these researchers specified a diffusion process for each implied volatility. Since implied volatilities can follow a stochastic dynamic, they had to be careful that the resulting pricing model did not allow for any arbitrage opportunities. This was done by setting specific conditions for the drift coefficient in the implied volatility process.

The drawback to these various approaches is that they result in a problem of high dimension; an empirical analysis of the implied volatility surface dynamic can help scale the number of dimensions down.

Cont, Da Fonseca, and Durrleman (2002) used the Principal Component Analysis (PCA hereafter) method developed by Cont and Da Fonseca (2002) (see next section) to determine a limited number of factors to model the stochastic movement of the implied volatility surface. They developed a linear factor model for the implied volatility logarithm, using mean-reversion functions for the factors. This model gives a simple estimation of parameter values based on historical data.

\footnotetext{
${ }^{2}$ This includes traditional models like those developed by Black and Scholes (1973), Merton (1973), and Cox and Ross (1976) as well as the local volatility models developed by Dupire (1994), Derman and Kani (1994a), and Rubinstein (1994).
} 
Daglish, Hull, and Suo (2007) addressed the problem in the same way as Schönbucher but they chose to model the implied variance (square of the implied volatility). Their work led to the following equation:

$d \sigma_{i m p}^{2}(T, K)=u(T, K) d t+\gamma(T, K) d W_{t}+\sigma_{i m p}^{2}(T, K) \sum_{i=1}^{N} v_{i}(T, K) d Z_{i}$

They then outlined the non-arbitrage conditions that the diffusion parameters must meet, and examined the effect that these conditions have on the observed implied volatility surface. The resulting general equation models implied volatility surface movements through certain factors (which could be correlated with the underlying asset), which were selected using an approach similar to the PCA and which meet the non-arbitrage conditions.

These latter two studies show the importance of an empirical study of the implied volatility surface dynamic. The following section discusses the main focuses of research in this area.

\subsection{Implied Volatility Surface Dynamics}

In order to develop market models, researchers must be able to identify the number and form of shocks that can cause the implied volatility surface to change over time and space given the possible states of the underlying asset.

The main studies of implied volatility surface dynamics use a PCA, which is a classic data reduction method. These studies looked either at the implied volatility term structure (Zhu \& Avellaneda, 1997; Fengler, Härdle, \& Schmidt, 2002; Sylla \& Villa, 2000) or at the smile (Skiadopoulos, Hodges, \& Clewlow, 1999; Alexander, 2001). Meanwhile, Kamal and Derman (1997), Skiadopoulos, Hodges, and Clewlow (1999), and Mixon (2002) used the same approach, but in a vector form, for the entire surface. Some researchers have developed variations of the PCA in order to account for specific features of the implied volatility surface. For instance, Ané and Labidi (2001) used an Independent Component Analysis to obtain components that are easy to interpret. Fengler, Härdle, and Villa (2003) jointly decomposed maturity groups using a Common PCA to account for the interdependence between the exercise price and maturity dimensions. Fengler, Härdle, and Mammen (2007) drew from the degenerating nature of the implied volatility surface to develop a semi-parametric factor model to bring the surface into a finite-dimension function space. Along the same lines, Cont and Da Fonseca (2002) and Kermiche (2008) used a functional form extension of the traditional PCA.

The advantage of the PCA is that the entire surface (or curve in the case of the term structure and smile) can be modelled using just a few variables, thereby reducing the size of the risk space. The above studies showed that two or three factors can generally be used to model between $60 \%$ and $95 \%$ of the total implied volatility surface variance. The first factor represents the curve's parallel movements, the second represents its slope, and the third represents its curvature.

Consequently an options pricing model must contain three or four factors to be consistent with the smile: one factor for the underlying asset and two or three factors for the implied volatility. Researchers including Cont and Da Fonseca (2002) and Kermiche (2008) have suggested using a mean-reversion process like Ornstein-Uhlenbeck to model volatility factors.

\section{SYNTHESIS AND FUTURE RESEARCH}

The table below offers a synthetic view of the advantages and disadvantages of each family of models. In the light of this synthesis, we can see that both jump diffusion models and stochastic volatility models have several advantages and disadvantages: jump diffusion models have good performances for short-term options but do not account for changes in the volatility, whereas stochastic volatility models allow volatility to move but are not very effective at pricing short-term options. By combining the two family models, we can greatly improve their performances. Nonetheless, these models fail to retain the market completeness and thus ignore important information offered by highly liquid standard options. Local volatility models overtake this shortfall and offer simple to use pricing tools. Unfortunately, the deterministic version of these models poorly performs in dynamic hedging. 
The stochastic local volatility models, as well as the market models offer interesting alternative to the previous models. Both use the information available on the highly liquid standard options market and present good performances on dynamic hedging. These models seem to be the most interesting ones to develop in the near future. To this end, and especially in times of excessive volatility on the markets, it seems important for researchers to focus on the empirical study of the volatility, and more precisely on the interactions between its different dimensions. This will help practitioners better forecast and hedge the excess volatility arising during crises.

\begin{tabular}{|l|l|l|}
\hline \multicolumn{1}{|c|}{ Family Models } & \multicolumn{1}{c|}{ Advantages } & \multicolumn{1}{c|}{ Disadvantages } \\
\hline Black-Scholes Model & $\begin{array}{l}\text { Straightforward method for pricing and } \\
\text { hedging derivatives }\end{array}$ & $\begin{array}{l}\text { Non-realistic assumptions: } \\
\text { Constant volatility } \\
\text { Normal distribution of the } \\
\text { underlying's returns }\end{array}$ \\
\hline Jump Diffusion Models & $\begin{array}{l}\text { Generate volatility smile and skew } \\
\text { consistent with market observations; } \\
\text { Outperform stochastic volatility models for } \\
\text { short-term options; } \\
\text { Better account for skewness and kurtosis in } \\
\text { the distribution of returns }\end{array}$ & $\begin{array}{l}\text { No simple equation to calculate option } \\
\text { prices; } \\
\text { Do not account for the change in the level } \\
\text { and shape of implied volatility over time }\end{array}$ \\
\hline Stochastic Volatility Models & $\begin{array}{l}\text { Different smile and skew shapes can be } \\
\text { obtained depending on the correlation and } \\
\text { the parameters used for the volatility } \\
\text { process }\end{array}$ & $\begin{array}{l}\text { Unrealistic correlation needed to obtain } \\
\text { market skew; } \\
\text { Multi-factor models that are difficult to } \\
\text { use for dynamic hedging; } \\
\text { Not very effective at pricing short-term } \\
\text { options }\end{array}$ \\
\hline Local Volatility Models & $\begin{array}{l}\text { Easy to use; } \\
\text { Resulting prices are consistent with liquid } \\
\text { options on the same underlying; } \\
\text { Retains the market completeness of the } \\
\text { Black-Scholes model }\end{array}$ & $\begin{array}{l}\text { Highly unstationary implied volatility; } \\
\text { Skew disappear in the near future; } \\
\text { The deterministic models poorly perform } \\
\text { in dynamic hedging }\end{array}$ \\
\hline Implied Volatility Market & $\begin{array}{l}\text { Based on observed information; } \\
\text { Consistent with the smile; } \\
\text { Good performance for dynamic hedging }\end{array}$ & \begin{tabular}{l} 
High-dimension problems \\
\hline
\end{tabular} \\
\hline
\end{tabular}

\section{CONCLUSION}

The Black-Scholes model (1973) is a founding element of modern options theory, and continues to be widely-used today. However, several of its assumptions have been challenged, especially in light of the substantial discrepancies between the model's assumptions and what is actually seen in the market. These discrepancies are most visible in the implied volatility surface (which depends on the exercise price and option maturity), and a nonnormal density function for the underlying asset return.

Several models have been developed over the past decades to remedy the shortcomings of Black-Scholes. These models can be divided into three categories: (i) jump diffusion models, introduced by Merton in 1976 soon after the emergence of Black-Scholes; (ii) stochastic volatility models, introduced in the late 1980s and consisting mainly on the work done by Hull and White (1987), Stein and Stein (1991), and Heston (1993); and (iii) local volatility models developed by Dupire (1994), Derman and Kani (1994a), and Rubinstein (1994). Researchers have also studied improvements to and combinations of these different categories.

The advantage of these models is that they give smiles that are consistent with what is seen in the markets, and return density curves with a negative skewness and excess kurtosis. However their drawbacks are that their parameters are often difficult to estimate (requiring unrealistic values), and that they can give options price and volatility smile movements that are not in line with market data-making them unsuitable for dynamic asset management.

A new family of models, called market models, has recently emerged; their name comes from the fact that they describe a variable (implied volatility) that can be estimated directly from options prices observed in the 
market. These models view highly-liquid options prices as market data, and use them to price illiquid and exotic options. In order to refine these models, empirical studies have been carried out to identify the shock factors that can affect these variables and the dynamics of these factors. Early research has focused on the term structure and the implied volatility smile, generally ignoring the interactions between these two dimensions. Few empirical studies have been carried out so far on the implied volatility surface as a whole, and these interactions still need to be explored. This is certainly an important area to develop, in order to offer the tools for implementing market models, which are definitely the most promising way for future research.

\section{AUTHOR INFORMATION}

Lamya Kermiche, Associate professor of Finance at Grenoble Ecole de Management, 12 rue Pierre Sémard, 38003 Grenoble cedex 01. Tél. + 33 (0)4 767065 34. Fax + 33 (0)4 767060 99. E-mail: Lamya.kermiche@ grenoble$\underline{\text { em.com }}$

\section{REFERENCES}

1. Alexander, C. (2001). Principal component analysis of volatility smiles and skews. EFMA 2001 Lugano Meetings. (Working Paper in Finance 2000-10). University of Reading.

2. Alexander, C., \& Nogueira, L. M. (2004). Hedging with stochastic local volatility. (ISMA Centre Discussion Papers in Finance 2004-10).

3. Andersen, L., \& Andreasen, J. (2000). Jump-diffusion processes: Volatility smile fitting and numerical methods for option pricing. Review of Derivatives Research, 4, 231-262.

4. Andersen, T. G., Benzoni, L., \& Lund, J. (2002). An empirical investigation of continuous-time equity return models. Journal of Finance, 57(3), 1239-1284.

5. Ané, T., \& Labidi, C. (2001). Implied volatility surfaces and market activity over time. Journal of Economics and Finance, 25(3).

6. Bakshi, G., Cao, C., \& Chen, Z. (1997). Empirical performance of alternative option pricing models. Journal of Finance, 52(5), 2003-2049.

7. Bakshi, G., Cao, C., \& Chen, Z. (2000). Do call prices and the underlying stock always move in the same direction? Review of Financial Studies, 13(3), 549-584.

8. Ball, C. A., \& Torous, W. N. (1985). On jumps in common stock prices and their impact on call option pricing. Journal of Finance, 40(1), 155-173.

9. Bates, D. S. (1996). Jumps and stochastic volatility: Exchange rate processes implicit in deutsche mark options. Review of Financial Studies, 9(1), 69-107.

10. Black, F., \& Scholes, M. (1973). The pricing of options and corporate liabilities. Journal of Political Economy, 81(3), 637-654.

11. Brace, A., Goldys, B., Klebaner, F., \& Womersley, R. (2001). Market model of stochastic volatility with applications to the BGM model. (Working paper S01-1). Department of Statistics, University of New South Wales.

12. Cont, R., \& Da Fonseca, J. (2002). Dynamics of implied volatility surfaces. Quantitative Finance, 2(1), 4560.

13. Cont, R., Da Fonseca, J., \& Durrleman, V. (2002). Stochastic models of implied volatility surfaces. Economic Notes: Review of Banking, Finance and Monetary Economics, 31(2), 361-377.

14. Daglish, T., Hull, J., \& Suo, W. (2007). Volatility surfaces: Theory, rules of thumb, and empirical evidence. Quantitative Finance, 7(5), 507-524.

15. Das, S. R. (2002). The surprise element: Jumps in interest rates. Journal of Econometrics, 106(1), 27-65.

16. Das, S. R., \& Foresi S. (1996). Exact solutions for bond and option prices with systematic jump risk. Review of Derivatives Research, 1(1), 7-24.

17. Das, S. R., \& Uppal, R. (1998). How diversified are internationally diversified portfolios: Time-variation in the covariances between international returns. Canadian Investment Review, Spring, 7-11.

18. Derman, E., \& Kani, I. (1994a). The volatility smile and its implied tree. Goldman Sachs, Quantitative Strategies Research Notes.

19. Derman, E., \& Kani, I. (1994b). Riding on a smile. Risk, 7(2), 32-39. 
20. Derman, E., \& Kani, I. (1998). Stochastic implied trees: Arbitrage pricing with stochastic term and strike structure of volatility. International Journal of Theoretical and Applied Finance, 1(1), 61-110.

21. Derman, E., Kani, I., \& Chriss, N. (1996). Implied trinomial trees of the volatility smile. Journal of Derivatives, 4(2), 7-22.

22. Dumas, B., Fleming, J., \& Whaley, R. E. (1998). Implied volatility functions: Empirical tests. Journal of Finance, 53(6), 2059-2106.

23. Dupire, B. (1993a). Pricing and hedging with smiles. (Working paper). Paribas.

24. Dupire, B. (1993b). Arbitrage pricing with stochastic volatility. (Working paper). Paribas.

25. Dupire, B. (1994). Pricing with a smile. Risk, 7(1), 18-20.

26. Dupire, B. (1996). A unified theory of volatility. (Working paper).

27. Fengler, M., Härdle, W., \& Schmidt, P. (2002). Common factors governing VDAX movements and the maximum loss. Journal of Financial Markets and Portfolio Management, 16(1), 16-29.

28. Fengler, M., Härdle, W., \& Villa, C. (2003). The dynamics of implied volatilities: A common principal component approach. Review of Derivatives Research, 6(3), 179-202.

29. Fengler, M., Härdle, W., \& Mammen, E. (2007). A semiparametric factor model for implied volatility surface dynamics. Journal of Financial Econometrics, 5(2), 189-218.

30. Fleming, J., Ostdiek, B., \& Whaley, R. E. (1995). Predicting stock market volatility: A new measure. Journal of Futures Markets, 15(3), 265-302.

31. Heston, S. L. (1993). A closed-form solution for options with stochastic volatility with applications to bond and currency options. Review of Financial Studies, 6(2), 327-343.

32. Hull, J. C., \& White, A. (1997). The pricing of options on assets with stochastic volatilities. Journal of Finance, 42(2), 281-300.

33. Kallsen, J., \& Krühner, P. (2014). On a Heath-Jarrow-Morton approach for stock options. Finance and Stochastics, forthcoming.

34. Kamal, M., \& Derman, E. (1997). The patterns of change in implied index volatilities. Goldman Sachs, Quantitative Strategies Research Notes.

35. Kermiche, L. (2008). Une modélisation de la surface de volatilité implicite par processus à sauts. Finance, 29(2), 57-101.

36. Le, T. (2005). Stochastic market volatility models. Applied Financial Economics Letters, 1, 177-188.

37. Ledoit, O., Santa-Clara, P., \& Yan, S. (2002). Relative pricing of options with stochastic volatility. (UCLA Finance Working Paper 9-98).

38. Merton, R. (1976). Option pricing when underlying stock returns are discontinuous. Journal of Financial Economics, 3(1-2), 125-144.

39. Mixon, S. (2002). Factors explaining movements in the implied volatility surface. Journal of Futures Markets, 22(10), 915-937.

40. $\quad$ Rubinstein, M. (1994). Implied binomial trees. Journal of Finance, 49(3), 771-818.

41. Schönbucher, P. J. (1999). A market model of stochastic implied volatility. Philosophical Transactions of the Royal Society. Series A, 357, 2071-2092.

42. Schweizer, M., \& Wissel, J. (2008a). Term structures of implied volatilities: Absence of arbitrage and existence results. Mathematical Finance, 18(1), 77-114.

43. Schweizer, M., \& Wissel, J. (2008b). Arbitrage-free market models for options prices: The multi-strike case. Finance and Stochastics, 12(4), 469-505.

44. Skiadopoulos, G. (2001). Volatility smile consistent option models: A survey. International Journal of Theoretical and Applied Finance, 4(3), 403-437.

45. Skiadopoulos, G., Hodges, S., \& Clewlow, L. (1999). The dynamics of the S\&P 500 implied volatility surface. Review of Derivatives Research, 3(3), 263-282.

46. Stein, E., \& Stein, C. (1991). Stock price distributions with stochastic volatilities: An analytical approach. Review of Financial Studies, 4(4), 727-752.

47. Sylla, A., \& Villa C. (2000). Measuring Implied volatility surface risk using principal components analysis. In J. Franke, W. Härdle, \& G. Stahl (Eds.), Measuring risk in complex stochastic systems, lecture notes in statistics (pp.131-147). Springer Verlag, New York.

48. Taylor, S. J., \& Xu, X. (1993). The magnitude of implied volatility smiles: Theory and empirical evidence for exchange rates. Review of Futures Markets, 13, 355-380. 
49. Zhu, Y., \& Avellaneda, M. (1997). An E-ARCH model for the term structure of implied volatility of FX options. Applied Mathematical Finance, 4(2), 81-100.

50. Zilber, A. (2007). A market model for stochastic smiles. (Working Paper). 